\title{
LES ARCHITECTES AU PORTUGAL : ENTRE LA VOCATION ET LA PROFESSION
}

\author{
Vera Borges, Manuel Villaverde Cabral
}

L'Harmattan | «Sociologie de l'Art »

2015/1 OPuS 25 \& 26 | pages 153 à 176

ISSN 0779-1674

ISBN 9782343090795

Article disponible en ligne à l'adresse :

http://www.cairn.info/revue-sociologie-de-l-art-2015-1-page-153.htm

\section{Pour citer cet article :}

Vera Borges, Manuel Villaverde Cabral, « Les architectes au Portugal : entre la vocation et la profession », Sociologie de l'Art 2015/1 (OPuS 25 \& 26), p. 153-176.

Distribution électronique Cairn.info pour L'Harmattan.

(C) L'Harmattan. Tous droits réservés pour tous pays.

La reproduction ou représentation de cet article, notamment par photocopie, n'est autorisée que dans les limites des conditions générales d'utilisation du site ou, le cas échéant, des conditions générales de la licence souscrite par votre établissement. Toute autre reproduction ou représentation, en tout ou partie, sous quelque forme et de quelque manière que ce soit, est interdite sauf accord préalable et écrit de l'éditeur, en dehors des cas prévus par la législation en vigueur en France. Il est précisé que son stockage dans une base de données est également interdit. 


\section{Les architectes au Portugal : entre la vocation et la profession}

Vera BORGES, DINÂMIA/CET-ISCTE - Institut Universitaire de Lisbonne - Institut des Sciences Sociales - Université de Lisbonne vera.borges@iscte.pt Manuel VILLAVERDE CABRAL, Institut des Sciences Sociales - Université de Lisbonne mvcabral@ics.ul.pt

Résumé : À partir des données recueillies lors d'une enquête sur les architectes au Portugal, nous essayons d'analyser pourquoi cette profession libérale basée sur l'obtention d'un diplôme universitaire et exercée sous le monopole de l'Ordre des architectes continue d'attirer plus de "vocations" que le marché ne peut en satisfaire. La conclusion la plus pertinente se trouve sur l'ethos de l'architecture, telle qu'elle est pratiquée aujourd'hui sur le terrain portugais, qui repose sur une tension récurrente entre "vocation" et "profession" en raison de sa forte dimension artistique qui, à son tour, la distingue des autres professions libérales.

Mots-clés: architectes - architecture - mondes artistiques vocation - profession 


\title{
Architects in Portugal: Between the vocation and the profession
}

\begin{abstract}
Based on the data collected during a survey of architects in Portugal, we analyze why this profession based on an academic degree and practiced under the monopoly of the Order of Architects continues to attract more "callings " than the market is capable of absorbing. The most relevant conclusion drawn from the analysis of the data is that the ethos of architecture, such as it is practised in Portugal, lies in a recurrent tension between "vocation " and "profession " as a result of its strong artistic dimension, which, in turn, distinguishes it from other liberal professions.
\end{abstract}

Keywords : architects - architecture - artistic worlds - vocation profession

\section{Arquitectos en Portugal: Entre la vocación y la profesión}

Resumo: A partir de los datos recogidos durante un estudio de arquitectos en Portugal, se trata de analizar por qué esta profesión basada en la obtención de un título universitario y se practica bajo el monopolio de la Orden de Arquitectos continúa atrayendo más "vocaciones" que el mercado no puede cumplir. La conclusión más relevante é que o ethos de la arquitectura, tal como se practica hoy en día en el caso portugués, en base a una tensión recurrente entre "vocación " y "profesión ", debido a su sólida dimensión artística, que, a su vez, a diferencia de otras profesiones.

Palabras-clave : arquitectos - arquitectura - mundos artísticos vocación - profesión 


\section{Les architectes au Portugal : entre la vocation et la profession}

\section{Introduction $^{1}$}

Cet article exploite les données recueillies lors d'une enquête menée auprès des architectes qui travaillent au Portugal. Un bref aperçu des réflexions que les résultats de l'enquête nous ont inspirées nous permet de décrire la centralité de la tension "vocation / profession " dans l'architecture et comment cette tension se manifeste sur ce terrain particulier.

En général, l'évolution de cette profession montre que le conflit juridictionnel qui oppose les architectes aux ingénieurs réside dans l'essor des sciences et des techniques survenu au XIX ${ }^{e}$ siècle, qui fournit alors à la construction un grand nombre de nouveaux matériaux ainsi que des procédés rigoureux et économiques permettant de les combiner. D'un point de vue chronologique, les architectes précèdent les ingénieurs civils et cumulent les compétences des deux professions jusqu'au début du $X X^{\mathrm{e}}$ siècle (Kostoff, 2000). À cette époque, la différenciation entre l'art et la technique (Francastel, 2000) mène à la certification de la profession d'ingénieur.

La reconnaissance de cette différence a posé des problèmes croissants à l'enseignement de l'architecture, symbolisé par l'École des Beaux-Arts de Paris (Egbert, 1980, 58-95). Toutefois, le processus de différenciation socioprofessionnelle qui a assuré l'ascension des ingénieurs civils n'a aboli ni l'autorité architecturale ni le "modèle Beaux-Arts" de transmission personnalisée des savoirs à travers la relation corporative entre le

\footnotetext{
1 Nous remercions vivement notre collègue Morgan Jouvenet pour ses remarques et suggestions pendant la rédaction finale de cet article.
} 
maître et l'apprenti. Inversement, aussi bien les architectes que les ingénieurs continuent aujourd'hui de subir la concurrence des " maîtres d'œuvres " pour les constructions ne requérant aucune solution complexe technique ni esthétique. L'avènement du Computer assisted design-CAD a influencé les pratiques de la conception architecturale (Champy, 1998, 2001); cependant, l'autorité conceptuelle reconnue aux architectes n'en a pas moins continué de se voir renforcée, au cours des dernières décennies, par le biais de la résurgence de l' " architecte-auteur ". Reconnu par son œuvre, "l'architecte-auteur » inspire les programmes de design et de "marque " des territoires, conçus dans des logiques politiques et de compétition économique entre pays et villes (Larson, 1993, 218-242) ${ }^{2}$.

Pour l'analyse du cas portugais ${ }^{3}$, nous avons repris l'idée présente dès les premiers ouvrages de sociologie, quand Max Weber publie Wissenschaft als Beruf et Politik als Beruf (Weber, 1919), et on constate que Weber utilise le terme Beruf tantôt comme synonyme de "profession ", dans le sens conventionnel du mot, tantôt comme synonyme de "vocation", dans le sens habituel que ce mot a dans les langues romanes (Weber, 2003), c'est-àdire dans le sens de "don ", "appel ", et même "charisme " dans l'acception religieuse du terme (Weber, 2002, 84ff). Nous emploierons le terme de "vocation", dans son sens latin, pour désigner une catégorie d'orientation occupationnelle pouvant ou non correspondre à une profession établie, mais à même de générer des activités professionnelles à partir de l'offre de ses savoirs scientifiques et pratiques sur le marché. II faut dire que l'argumentation sur «la science comme vocation et comme profession " développée par Weber peut s'appliquer en grande partie à l'art (Weber, 2003, 80-81). P.-M. Menger considère que dans les mondes de l'art toutes ces qualités sont la condition

\footnotetext{
${ }^{2}$ Voir " l'effet Guggenheim », à Bilbau (Ponzini, 2010) et, sur le cas portugais, la reconstruction d'une partie de la ville de Lisbonne a partir de l'Expo98, dans la perspective de créer une nouvelle " image de la ville » (Lynch, 1960).

${ }^{3}$ Le Portugal compte, proportionnellement au total de la population, plus du double d'architectes que la France et ce pour un marché proportionnellement moindre et en état de crise profonde depuis plusieurs années.
} 
préalable, à la fois à l'invention et à la satisfaction associées au travail artistique (Menger, 2005, 7-29). L'appel de ces activités créatives est d'ailleurs décrit dans la plupart des domaines artistiques.

C'est justement cet "appel» à double sens que nous désignons sous le terme de "vocation " et qui aide à comprendre au nom de quoi autant de jeunes se lancent dans cette profession et ce malgré les frictions entre l'offre et la demande existant sur le marché de l'architecture, et pourquoi ils y restent, contrairement aux licenciés en droit qui renoncent au barreau ou aux architectes français qui ne s'inscrivent pas à l'Ordre (Champy, 2001).

L'approche de P.-M. Menger (2009, 2012), montrant que les professionnels des arts sont jeunes, créatifs, hautement qualifiés, et plus motivés par les récompenses symboliques procurées par ces professions que par les gains financiers qu'ils peuvent en retirer, aide à comprendre certaines des caractéristiques que nous retrouvons chez les architectes portugais. Dans le même sens, E. Freidson fait allusion à la possibilité que l'art soit un «travail de vocation», par opposition au "travail aliéné » qui a pour fin le "gain matériel ", comme si "vocation " et "gain " étaient incompatibles (Freidson, 1986, pp. 441-442 ; Freidson, 1990). Les conclusions de leurs travaux aident à réfléchir sur le défi lancé au sociologue par les terrains artistiques. Dans ces terrains, la composante vocationnelle explique les gratifications symboliques que les architectes obtiennent, en dépit des obstacles matériels au plein exercice de l'architecture. Les architectes ont, en effet, la rare possibilité d'inscrire leur "marque" dans l'espace et le temps (Raynaud, 2001), d'où la dérive démiurgique qui marque l'enseignement de la discipline et dont on retrouve la trace dans certaines des réponses à notre enquête (voir Tableau 1).

Par ailleurs, la pluralité des acteurs en compétition sur ce marché, la multiplicité des statuts professionnels et l'hétérogénéité parmi les architectes ainsi que le développement de tâches multiples à l'intérieur et à l'extérieur des ateliers existent au Portugal et continuent d'exister en France (Moulin et Champy, 1993; Champy, 2001). De même, au Portugal, nous avons pu également 
constater que « la création dans ce domaine comme dans d'autres est le privilège de quelques-uns [et que] l'architecte, quand il n'est pas éliminé du processus de construction, est très souvent appelé à fournir des services sans gloire " (Moulin, 1973, 280). Néanmoins, on ne constate aucune diminution du nombre de "vocation", et ce sont justement ces "vocations" qui présentent un défi pour la sociologie.

\section{Les résultats ${ }^{4}$}

L'actuelle composition sociodémographique des architectes portugais est marquée par leur extrême jeunesse. Ce constat est, aujourd'hui, le même pour toutes les professions qui requièrent un diplôme universitaire, mais rares sont celles dans lesquelles le renouvellement a été si tardif et si accentué. Plus de la moitié des architectes sont âgés de 35 ans ou moins et l'Ordre reçoit 1000 inscriptions par an, c'est-à-dire un chiffre bien supérieur au nombre total d'architectes existant avant le 25 avril 1974. Ce profond renouvellement générationnel explique les problèmes

4 L'intérêt de notre enquête est d'abord de fournir une caractérisation sociologique des architectes portugais, à partir d'un échantillon important de personnes. Par comparaison, l'Ordre des Architectes organise en 1984 la première enquête qui obtient près de 560 réponses puis commande la deuxième en 1989 obtenant 760 réponses. Cette enquête a été réalisée au moyen d'un questionnaire fermé, à remplir par chaque informateur, et envoyé par courrier (après un pré-test) à tous les membres de l'Ordre des Architectes, hormis les stagiaires - soit 12. 632 individus. La soumission du questionnaire a eu lieu entre décembre 2005 et avril 2006 et l'on a reçu 3198 réponses valides. L'échantillon est représentatif de l'univers des architectes inscrits à l'Ordre et la marge d'erreur est de $1,73 \%$ pour un intervalle de confiance de $95 \%$. Selon les trois critères de contrôle utilisés (sexe, âge et zone géographique d'inscription à l'ordre), on n'a observé aucune déviation significative en ce qui concerne les deux derniers critères; on a toutefois constaté une surreprésentation des plus jeunes de l'ordre de $8 \%$ par rapport à la moyenne d'âge de l'univers des répondants. Cette déviation aurait pu être corrigée en utilisant un pondérateur mais nous avons choisi de ne pas le faire afin de ne pas perdre les avantages de cette surreprésentation involontaire qui illustre une plus grande mobilisation des jeunes et permet de connaître en détail cette tranche d'âge, dans laquelle se concentrent les problèmes auxquels se confronte généralement la profession. Le taux de réponse (près de $25 \%$ ) constitue également un indicateur de mobilisation des architectes. 
auxquels la profession doit faire face que les différentes modalités d'insertion dans la vie professionnelle, depuis l'accès à la profession jusqu'au plein exercice de l'architecture en libéral, en passant par les revenus perçus, ainsi que par les différentes attitudes à l'égard de l'architecture elle-même. Si les architectes hommes sont jeunes, les femmes le sont encore plus : $70 \%$ d'entre elles ont moins de 35 ans.

En effet, le second trait marquant de la composition sociologique de la profession est à mettre en corrélation avec le premier et réside dans sa féminisation accélérée. Les femmes constituent près de $35 \%$ des effectifs de la profession et plus de la moitié de chaque contingent annuel. La féminisation de l'architecture se fait à un rythme plus lent que celle des autres professions nécessitant un diplôme universitaire et les femmes architectes révèlent des parcours professionnels et des profils d'attitudes différents de ceux de leurs pairs masculins. Ces différences se manifestent déjà au niveau de leur parcours de formation, de leur taux d'activité durant la licence et de la façon dont elles obtiennent leur premier emploi et configurent un profil de formation plus scolaire que pratique.

En ce qui concerne l'exercice professionnel de l'architecture, les hommes exercent plus souvent en libéral alors que les femmes travaillent soit comme salariées soit en régime de prestation de services, et leur situation sur le marché du travail est toujours plus précaire que celle des hommes. Les différences existent également dans les domaines d'activités: les constructions les plus importantes occupent davantage les hommes qui soumettent deux fois plus de projets à des concours et sont pratiquement deux fois plus nombreux à gagner des prix. Elles cumulent moins d'activités et leurs revenus sont inférieurs.

Ces différences sont récurrentes et configurent des modèles professionnels et d'attitudes distincts: les femmes se montrent plus insatisfaites que les hommes des conditions dans lesquelles elles exercent l'architecture et, plus que les hommes, elles souhaitent que la profession aille vers plus de technique, de spécialisation, de réglementation et de protection de 
l'environnement. Les divergences demeurent quand on leur demande d'indiquer les problèmes auxquels se heurte la profession: tandis que les femmes désignent la précarité des conditions de travail et la concurrence provenant d'autres professions, les hommes se soucient davantage de la législation et de la bureaucratie qui entravent l'exercice professionnel.

En revanche, il n'existe aucun changement substantiel en ce qui concerne la distribution géographique: les architectes ne dérogent pas à une tradition, présente dans la formation de leur profession, qui fait qu'ils choisissent de s'installer à proximité des grands centres urbains et de décision, et demeurent encore plus concentrés dans les métropoles de Lisbonne et de Porto que la majorité des autres professionnels - on y compte près des deux tiers de la profession. Pas de grands changements non plus concernant les origines sociales des nouveaux architectes en dépit de la relative "démocratisation » de la formation supérieure due surtout à l'arrivée, sur le marché de l'enseignement, d'établissements privés octroyant, à partir des années 1980, des licences en architecture. Les parents des architectes enquêtés possèdent un "capital scolaire» deux fois plus élevé que la moyenne. Aujourd'hui, les diplômés d'établissements privés représentent $43 \%$ des architectes qui exercent, et tout semble indiquer que le renouvellement générationnel de la profession se fera de plus en plus grâce à ce type d'établissement.

\section{Une profession de nature artistique}

En ce qui concerne les habitudes culturelles des architectes, celles-ci sont d'autant plus pertinentes pour l'analyse que la dimension artistique de l'architecture fait appel au capital hérité et à l'acquis (Bourdieu, [1979] 2003) : d'un côté, les habitudes culturelles sont des formes de socialisation artistique et contribuent à l'éveil de la "vocation"; de l'autre, la familiarité avec le patrimoine architectural et artistique fait partie intégrante de l'enseignement de l'architecture et constitue une référence appropriable par le travail courant de l'architecte. Quand on compare les habitudes culturelles des architectes à celles du reste de la population portugaise, on constate que la visite régulière et 
même occasionnelle de musées ne concerne que $34 \%$ des Portugais, alors que $64 \%$ des architectes enquêtés déclarent se rendre au musée entre une à dix fois par an. On voit se confirmer I'hypothèse que les pratiques culturelles des architectes sont plus intenses et diversifiées que celles de la majorité de la population.

Outre le degré de fermeture socioculturelle révélé par les origines sociales et par les pratiques culturelles, il est très significatif que, malgré le renouvellement générationnel récent, la profession connaît un taux de reproduction interne, mesuré d'après le pourcentage d'architectes enfants et/ou parents proches d'architectes, supérieur à tout ce que l'on connaît de toutes les autres professions libérales (a titre indicatif, $25 \%$ des architectes ont au moins un parent proche ou éloigné qui exercent cette profession). Ce degré élevé de reproduction familiale aide à mieux comprendre la reproduction de l'ethos professionnel, ainsi que I'intériorisation des frustrations et la basse conflictualité entre les "nouveaux" et les "anciens" et ce, malgré les nombreux obstacles au plein exercice de la profession au Portugal. Les réseaux familiaux procurent également la concentration de ressources matérielles et symboliques favorables au succès professionnel. Néanmoins, ces facteurs constituent des obstacles au changement, concrètement à l'adaptation à un marché dont le rythme de croissance n'a pas suivi celui des effectifs professionnels, et auquel les architectes n'ont pas réussi à imposer leur pleine juridiction.

Nous devons signaler qu'au moment de l'enquête, la situation socioprofessionnelle des architectes au Portugal est le résultat de la combinaison de trois variables: nombre de professionnels (offre), volume du marché (demande) et compétence juridictionnelle, cette dernière étant, la variable la plus manipulable des trois. Selon les résultats de notre enquête, l'architecture demeure divisée entre un ethos esthétique, qui la place parmi les professions de nature artistique, caractérisées par une croissance exponentielle de l'offre, et un ethos technicoscientifique qui, à son tour, la place sur le chemin du 
professionnalisme fondé sur un diplôme d'enseignement supérieur.

Comme dans les professions dont l'exercice est soumis à l'obtention d'un titre universitaire, l'augmentation du nombre de diplômés en architecture a mené au prolongement de la formation et à une entrée plus tardive sur le marché du travail : fréquentation de stages obligatoires exigés par l'Ordre, poursuite des études en master et doctorat, inscription dans des formations post-graduées ou dans des cours de formation professionnelle. Ils sont près de $40 \%$ à prolonger ou à avoir prolongé ainsi leur formation.

Une moyenne élevée ${ }^{5}$ pour pouvoir entrer en Faculté, un parcours universitaire long et peu de débouchés professionnels, autant de caractéristiques qui contribuent à favoriser l'accès de candidats très motivés, détenteurs d'un capital culturel élevé et entretenant des liens familiaux avec la profession. Ces conditions contribuent à leur tour à la reproduction du profil social et de l'ethos professionnel rendant plus difficile tout changement sur le plan des études et de la profession. Ce n'est pas un hasard si la formation fournie par les études, notamment lorsqu'il s'agit de préparer les étudiants à leur vie professionnelle, a été considérée insuffisante par la majorité des enquêtés (62\%).

\section{Le déclin du modèle « Beaux-Arts "}

En compensation, la plupart d'entre les architectes ont travaillé pendant leurs études et la majorité ont exercé une activité en rapport avec l'architecture, ce qui leur aura assuré l'apprentissage de capacités que l'enseignement académique ne leur fournissait pas, facilitant ainsi leur intégration dans la profession et contribuant d'autant au développement futur de leur carrière. Toutefois, aujourd'hui la forte augmentation du nombre de postulants réduit cette possibilité. Privés d'une expérience que le

\footnotetext{
${ }^{5}$ Au Portugal, a titre d'exemple, en 2007, la note d'entrée du dernier élève admis en $1^{\text {ère }}$ année à la Faculté d'Architecture de l'Université technique de Lisbonne a été de 17,7 et celle du dernier candidat accepté en $1^{\text {ère }}$ année à la Faculté d'architecture de Porto de 18, 1.
} 
marché du travail leur procure de moins en moins au cours de leurs études, les très jeunes architectes fraichement diplômés ont plus de difficultés à entrer dans la profession.

En fait, l'enseignement du modèle "Beaux-Arts" s'adapte difficilement à la massification scolaire. La moitié des architectes reconnaissent qu'ils sont entrés dans la profession sous l'influence d'un professeur ou de quelqu'un (un parent, un ami ou camarade) directement lié à l'architecture. Le degré élevé d'autoreproduction qui caractérise la profession se voit confirmé tout comme d'ailleurs le déclin du modèle "Beaux-Arts ". Celui-ci était basé sur une relation initiatique entre le maître et l'apprenti qui articulait les dimensions vocationnelle et professionnelle de l'architecture. Le recours à des réseaux de connaissance dans les professions artistiques est d'ailleurs un moyen de réduire les coûts de recrutements (Menger, 1999, 2009) marqués par l'urgence, la précarité et la petite dimension des projets d'architecture au Portugal.

Ainsi, le "penchant pour l'architecture ", le " don pour le dessin», I' " intérêt pour l'art ", la "fréquentation assidue d'architectes" et même les "visites de monuments et de musées " apparaissent comme des motivations assumées par la grande majorité des architectes, ce qui vient confirmer l'idée de "vocation artistique». Néanmoins, les motifs concrets qui ont amené au choix des études $d$ 'architecture, une fois la dimension vocationnelle contrôlée, indiquent les motivations associées aux professions réputées pour leur "prestige social» $(29,6 \%)$ et leurs "nombreux débouchés professionnels" (19\%), ou bien alors pour des motivations directement liées au milieu social (31,6 \% à l'influence familiale et $19 \%$ à l'influence des amis). Quant à la dimension financière, elle est clairement mise de côté par la très grande majorité (ils sont seulement $4 \%$ à considérer cette dimension importante), ce qui constitue une anticipation des rémunérations faibles dans cette profession.

Le passage de la formation à la profession d'architecte est loin d'être linéaire. Les modalités de l'exercice professionnel sont extrêmement complexes et présentent un éventail flexible de 
multiples occupations. En réalité les architectes qui se consacrent exclusivement à une seule modalité d'exercice professionnel sont une minorité. La majeure partie cumule plus d'une modalité, et la diversification d'activités présente une double dimension: externe quand les activités exercées sortent du champ de l'architecture, par exemple l'enseignement, mais également interne pour ce qui relève des différents domaines de l'architecture auxquels se consacrent la majorité, révélant un degré de spécialisation très peu développé.

On compte 2.145 situations de cumul englobant $53 \%$ de l'univers enquêté; les combinatoires les plus nombreuses sont celles concernant les architectes qui ne déclarent aucune activité principale (16\%) et parmi lesquels prédominent des architectes plus âgés, probablement à la retraite, mais également de très jeunes individus qui cumulent surtout des activités en tant que "prestataires de services ". C'est dans ce même groupe que nous avons repéré le plus grand nombre d'architectes inactifs mais inscrits à l'Ordre.

Le deuxième groupe pratiquant le plus le cumul est celui des fonctionnaires de l'administration centrale et locale responsables de $18 \%$ du total des cumuls avec l'exercice indépendant de l'architecture. Quant aux autres architectes salariés, ils sont responsables de près de $10 \%$ des cumuls en libéral. Enfin, les architectes dont l'activité principale est la «prestation de services " comptent pour $8 \%$ des cumuls en libéral; si l'on inclut tous les autres types de cumuls, ces "prestataires de services» sont responsables de près de $30 \%$ des formes de cumul, prolongeant ainsi l'univers fragmenté dont nous avons parlé plus haut.

L'idéal-type de l'architecture comme profession libérale exercée en exclusivité est loin de correspondre à la réalité : ils sont moins de $40 \%$ à être à leur compte et à faire de l'architecture leur activité principale, sans compter que beaucoup cumulent avec une ou d'autres activités. Un tiers des architectes exerce la profession à titre principal comme salarié dans l'administration centrale, locale ou régionale, au service d'autres architectes ou 
d'autres entreprises ce qui n'empêche pas la majorité d'entre eux de cumuler des activités, notamment en régime libéral. Plus élevé encore est le pourcentage de ceux qui exercent leur activité principale en tant que prestataires, auprès d'autres architectes ou de professionnels et d'entreprises d'autres types les rémunérant à la tâche par le biais d'un système de "recibos verdes " (reçus verts), et qui cumulent ce statut avec l'exercice de la profession en libéral.

Les fluctuations présentes dans les réponses des enquêtés reflètent l'indéfinition, la superposition, la variation ainsi que la précarité des statuts professionnels (Menger, 2009). Les conditions d'exercice de l'architecture et de statut des architectes relativisent l'usage de la notion de profession principale. Ces tendances sont en fait des stratégies d'adaptation dictées par I' " offre excessive " d'architectes et fonctionnent pour beaucoup de professionnels comme un palliatif au sous-emploi des compétences acquises. Reste à savoir ce que font exactement les architectes portugais.

\section{L'importance de la conception des projets et la concentration de ressources}

Profession historiquement associé aux mondes des arts, l'architecture continue de lutter pour exercer la pleine juridiction sur la construction des bâtiments. Callon a déclaré que "l'architecte n'existe pas" (Callon, 1996, 29, apud Raynaud, 2001, 460), suggérant que les architectes ne possèdent aucune expertise spécifique qui ne soit négociée avec d'autres professionnels. La conception architecturale ne serait que le simple produit de la négociation entre les membres d'un réseau ouvert de partenaires. En réponse, Raynaud démontre dans un article sur les compétences des architectes que " la conception » continue d'être le noyau dur de l'expertise professionnelle de l'architecte (Raynaud, 2001, 451), comme Moulin (1973) et Champy (2001) l'avaient déjà remarqué. Raynaud conclut que les contenus de l'expertise architecturale résident dans la capacité d'analyser les contraintes de la localisation du projet, de formuler 
une réponse architectonique satisficing (Simon, 1981) à ces contraintes et d'imaginer les réactions des utilisateurs du bâtiment virtuel (Raynaud, 2001, 451-455).

En effet, la plupart des architectes portugais déclarent que leur principal domaine de travail est celui de conception/étude et des projets, même si certains ne seront jamais exécutés, comme c'est d'ailleurs le cas des milliers de projets jamais construits soumis régulièrement à concours par des architectes du monde entier ${ }^{6}$. En revanche, seule une minorité d'architectes dit s'occuper de tâches de gestion, de direction et d'inspection des travaux comme activité principale, démontrant ainsi que la conception précède la construction et en est autonome. Quant aux architectes développant des activités liées à l'enseignement et à la recherche, ils ne renoncent pas pour autant à développer des activités liées directement à l'architecture ni à s'installer en libéral ; ils illustrent en ce sens l'idée $d^{\prime}$ " emplois refuge (...) cumulés avec l'activité à vocation créatrice » (Menger, 2005, 16).

Dans le domaine de la conception, I'habitation unifamiliale demeure de loin le champ d'intervention des architectes portugais le plus important (presque $60 \% s^{\prime} y$ consacrent). En ce qui concerne l'habitation plurifamiliale, ils sont moins de $30 \%$ à s'y consacrer, un pourcentage semblable à celui des professionnels qui élaborent des projets de bâtiments publics et d'équipements collectifs. II s'agit là de marchés importants, mais qui concernent l'activité principale de seulement $30 \%$ des architectes et qui indiquent une forte concentration des ressources que l'on constate également dans les projets soumis à concours.

Ces indicateurs de concentration de ressources (Merton, 1988 ; Menger, 2009) se reflètent dans les rémunérations que les architectes perçoivent de leur activité principale et qui se situent en moyenne au-dessous de 2000 euros bruts par mois. Ils ne sont

\footnotetext{
${ }^{6}$ Voir par exemple les projets d'avant-garde conçus dans l'ancienne Russie soviétique, qui n'ont vu le jour qu'après avoir été récupérés plus tard par l'Occident (Tafuri et al., 1972).
} 
que $10 \%$ à déclarer des revenus mensuels supérieurs à 3.000 euros. Quant aux revenus provenant des différentes activités cumulées, loin de réduire les inégalités de revenus au sein de la profession, ils les aggravent - malgré le grand nombre d'architectes qui cumulent activités professionnelles, seuls $36 \%$ déclarent retirer des revenus de leurs activités secondaires.

Le croisement entre les revenus provenant de l'activité principale et ceux correspondant aux autres activités accumulées par les professionnels révèle l'existence de deux groupes distincts de dimensions différentes: d'une part, un grand nombre d'architectes qui pratiquent le cumul d'activités pour compléter des revenus inférieurs à la moyenne procurés par leur activité principale et, d'autre part, une petite minorité de professionnels qui perçoivent des revenus au-dessus de la moyenne et qui "cumulent par passion" d'autres activités, elles aussi bien rémunérées, d'où une forte corrélation positive entre les deux rémunérations qui se traduit donc par l'augmentation de la concentration des revenus au sein de la profession.

Les deux tiers des architectes portugais exercent en libéral, pour moitié en exclusivité et pour moitié en cumul, ce qui justifie pleinement que l'on doive mieux connaître les conditions dans lesquelles ils travaillent et les problèmes auxquels ils sont confrontés. On distingue deux grands groupes, selon que les professionnels travaillent dans un atelier (60\%) ou à domicile (40\%); cette distinction et celle qui sépare les professionnels exerçant exclusivement en libéral de ceux qui pratiquent le cumul se recoupent en grande partie. On constate le même phénomène de coïncidence en ce qui concerne la distinction entre ceux qui exercent l'architecture uniquement dans le département où ils résident et dans les zones limitrophes et ceux qui travaillent dans le pays tout entier (50\%) et même à l'étranger (7\%).

Il existe donc deux profils : d'une part, ceux qui exercent partout dans le pays et à l'étranger et exclusivement en libéral dans leurs ateliers et, d'autre part, ceux qui exercent seulement dans le département où ils résident ou à proximité et cumulent plusieurs activités qu'ils exercent à domicile; signalons que dans la région 
Sud, il existe plus d'architectes qui appartiennent au premier profil que dans la région Nord, et ce en raison de la dimension des marchés respectifs. Les principaux clients des architectes indépendants sont les particuliers, ce qui confirme la tendance aux petites commandes, puis ensuite viennent les PME qui suivent la même tendance. Les architectes qui travaillent pour de gros clients, comme l'État et les promoteurs immobiliers, sont peu nombreux, concentrant ainsi les opportunités dans un segment restreint de la profession.

\section{L'importance de l'ethos vocationnel}

Ce portrait sociologique de la profession laisse penser que les architectes portugais ne sont pas divisés en ce qui concerne leurs aspirations vocationnelles - ce qui, à leurs yeux, distingue l'architecture des autres professions. Le bien-être symbolique constitue la principale dimension de leur identité et les gratifications symboliques sont évaluées de façon très positive contrairement aux motivations matérielles (les revenus de la profession) que la grande majorité juge de façon négative. La présence de ces deux composantes dans la dimension dominante de l'identité des architectes portugais confirme la thèse d'une forte tension entre vocation et profession qui caractérise l'exercice de l'architecture.

La deuxième dimension est celle de l'auto-estime professionnelle qui offre, elle aussi, une image marquée par l'incertitude: si la "fierté d'être architecte" fait la quasi-unanimité chez les répondants, d'autres composantes sont évaluées de façon négative, en particulier l'image publique que les architectes estiment avoir.

La troisième dimension reflète également la façon négative dont la majorité des architectes perçoit l'image sociale de la profession. Il existe trois autres dimensions de moindre poids: I'une reflète des préoccupations liées à la régulation et en appelle à l'intervention de l'Ordre et des pouvoirs publics, ce qui révèle un sentiment défensif à l'égard de certains problèmes de la profession; une autre se préoccupe du changement technique et 
organisationnel et laisse ainsi deviner une inquiétude concernant l'avenir; enfin, dans la dernière dimension, émerge, en contrepartie, une orientation positive au changement professionnel.

\section{Tableau $n^{\circ} 1$}

Dimensions initiales sélectionnées pour l’analyse de clusters

\begin{tabular}{|l|l|l|l|}
\hline & $\begin{array}{l}\text { Satisfaction } \\
\text { professionnelle } \\
(19,0 \%)\end{array}$ & $\begin{array}{l}\text { Réalisation } \\
\text { vocationnelle } \\
(8,86 \%)\end{array}$ & $\begin{array}{l}\text { Orientation au } \\
\text { changement } \\
(6,61 \%)\end{array}$ \\
\hline $\begin{array}{l}\text { La société voit } \\
\text { l'architecture comme un } \\
\text { luxe }\end{array}$ &, 583 & & \\
\hline $\begin{array}{l}\text { La plupart des citoyens } \\
\text { ne savent pas ce que font } \\
\text { les architectes }\end{array}$ &, 581 & & \\
\hline $\begin{array}{l}\text { Les ingénieurs sont } \\
\text { considérés plus } \\
\text { importants que les } \\
\text { architectes }\end{array}$ & 565 & & \\
\hline $\begin{array}{l}\text { Les clients ne } \\
\text { reconnaissent pas sa } \\
\text { vraie valeur au travail des } \\
\text { architectes }\end{array}$ & -551 & & \\
\hline $\begin{array}{l}\text { Je suis satisfait des } \\
\text { rémunérations que je } \\
\text { perçois en tant } \\
\text { qu'architecte } \\
\text { lesquelles j'exerce } \\
\text { l'architecture }\end{array}$ & -511 & & \\
\hline & & & \\
\hline
\end{tabular}




\begin{tabular}{|l|l|l|l|}
\hline $\begin{array}{l}\text { L'argent que les } \\
\text { architectes gagnent ne } \\
\text { suffit pas à payer leurs } \\
\text { frais }\end{array}$ & ,499 & & \\
\hline $\begin{array}{l}\text { Les architectes ne sont } \\
\text { pas suffisamment unis } \\
\text { pour imposer la } \\
\text { résolution de leurs } \\
\text { problèmes }\end{array}$ & $\quad, 488$ & & \\
\hline $\begin{array}{l}\text { L'image générale des } \\
\text { architectes dans la } \\
\text { société est positive }\end{array}$ & & & \\
\hline $\begin{array}{l}\text { Si c'était à refaire, je } \\
\text { ferais à nouveau le choix } \\
\text { de l'architecture }\end{array}$ &,- 452 & & \\
\hline $\begin{array}{l}\text { Je regrette souvent } \\
\text { d'être architecte }\end{array}$ & &, 717 & \\
\hline $\begin{array}{l}\text { La profession } \\
\text { d'architecte correspond } \\
\text { à ma vocation }\end{array}$ & &, 601 & \\
\hline $\begin{array}{l}\text { J'ai confiance dans le } \\
\text { futur de l'architecture }\end{array}$ & &, 656 & \\
\hline $\begin{array}{l}\text { Avec la modernisation du } \\
\text { pays, les architectes } \\
\text { seront de plus en plus } \\
\text { nécessaires }\end{array}$ & &, 557 & \\
\hline $\begin{array}{l}\text { Je suis fier d'appartenir à } \\
\text { la classe des architectes }\end{array}$ & & & \\
\hline $\begin{array}{l}\text { En général je me sens } \\
\text { bien avec moi-même }\end{array}$ & & & \\
\hline $\begin{array}{l}\text { Dans l'exercice de leur } \\
\text { profession, les } \\
\text { architectes doivent se } \\
\text { spécialiser dans un } \\
\text { domaine précis }\end{array}$ & & & \\
\hline
\end{tabular}




\section{Actuellement, I'architecture est une profession essentiellement technique qui répond à des besoins de la société}

Sur la base d'une série d'analyses factorielles en composantes principales qui a sélectionné les 18 variables présentes dans le tableau 1 , a été réalisée une analyse de clusters $^{7}$ qui montre que les architectes portugais se divisent en cinq groupes, selon les axes de la satisfaction, de la réalisation vocationnelle et de l'orientation au changement, à savoir, par ordre de dimension : $31 \%$ de mécontents; $23 \%$ d'innovateurs; $21 \%$ de conservateurs; $14 \%$ de non résignés et $11 \%$ de déçus. II faut signaler qu'aucun de ces groupes ne révèle de degré de satisfaction professionnelle positif et se différencient à peine par l'intensité de leur insatisfaction. En résumé : $42 \%$ des architectes portugais sont mécontents ou même déçus des conditions dans lesquelles ils exercent leur profession tandis que $14 \%$ d'entre eux (les non résignés) ne baissent pas les bras devant l'état actuel des choses et sont à la recherche de solutions innovantes. Cela signifie que plus de la moitié des architectes, surtout parmi les jeunes et les femmes, est loin d'avoir réussi jusqu'à présent. En revanche, l'enquête révèle que près de la moitié des architectes (44\%), surtout parmi les plus âgés et les hommes, ont "réussi »: satisfaction professionnelle au-dessus de la moyenne et gratification vocationnelle élevée. Ce sont eux qui composent le groupe des novateurs (23\%) et des conservateurs (21\%): la principale dimension qui les distingue est l'orientation au changement : positive pour les premiers, ayant étudié en majorité

\footnotetext{
${ }^{7}$ Ces profils ont été obtenus sur la base d'une analyse hiérarchique de clusters, afin d'identifier des groupes d'architectes ayant des caractéristiques relativement différentes dans les dimensions testées. Nous avons choisi de retenir les cinq plus grands groupes de manière à ce que le plus petit d'entre eux ne représente pas moins de $10 \%$ des enquêtés.
} 
dans le privé, elle est négative pour les deuxièmes qui sont également plus âgés.

\section{Graphique 1 \\ Profil des architectes}

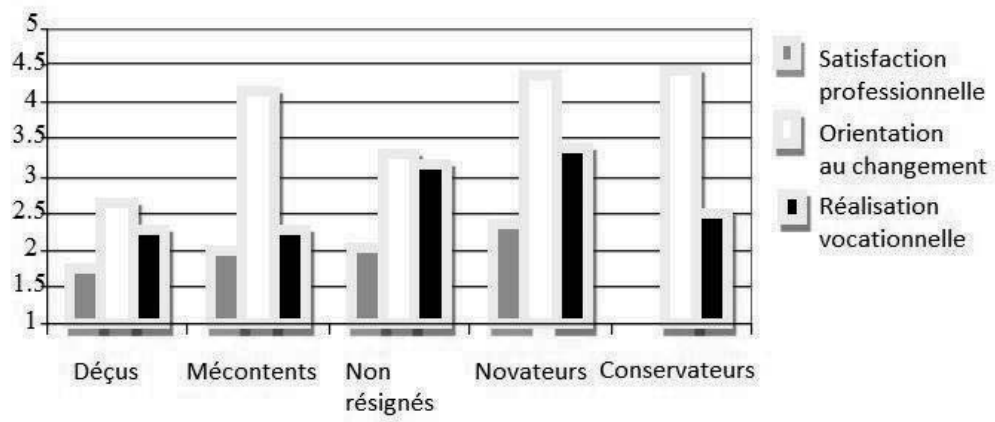

L'examen des facteurs qui, selon les architectes portugais, contribuent le plus à la réussite professionnelle révèle que l'indicateur le plus favorable est la position atteinte dans la hiérarchie socioprofessionnelle où se combinent la modalité principale de l'exercice de la profession et les revenus perçus. Au sommet de cette hiérarchie, se trouve l'exercice de l'architecture en libéral, en qualité de membre d'une société d'architectes; ensuite, viennent les professionnels libéraux à titre individuel suivis des salariés et juste après des «prestataires» et, pour finir, ceux qui déclarent ne pas avoir d'activité principale.

D'après notre modèle interprétatif, l'explication du succès professionnel est contingente, dans la mesure où le modèle ne rend compte que de $18 \%$ de la variance expliquée. Le modèle interprétatif est basé sur les propriétés sociodémographiques et professionnelles qui pourraient contribuer à expliquer le succès d'une carrière et a dégagé, à travers une régression linéaire, les indicateurs présents sur le schéma ainsi que leur poids respectif. Ensuite, lorsqu'on a placé le succès professionnel au centre du schéma, celui-ci a contribué à expliquer les sentiments de satisfaction sociale et de satisfaction matérielle, qui ne se recoupent pas totalement, étant donné la disjonction observée 
entre l'architecture comme profession et l'architecture comme vocation.

\section{Diagramme du modèle interprétatif}

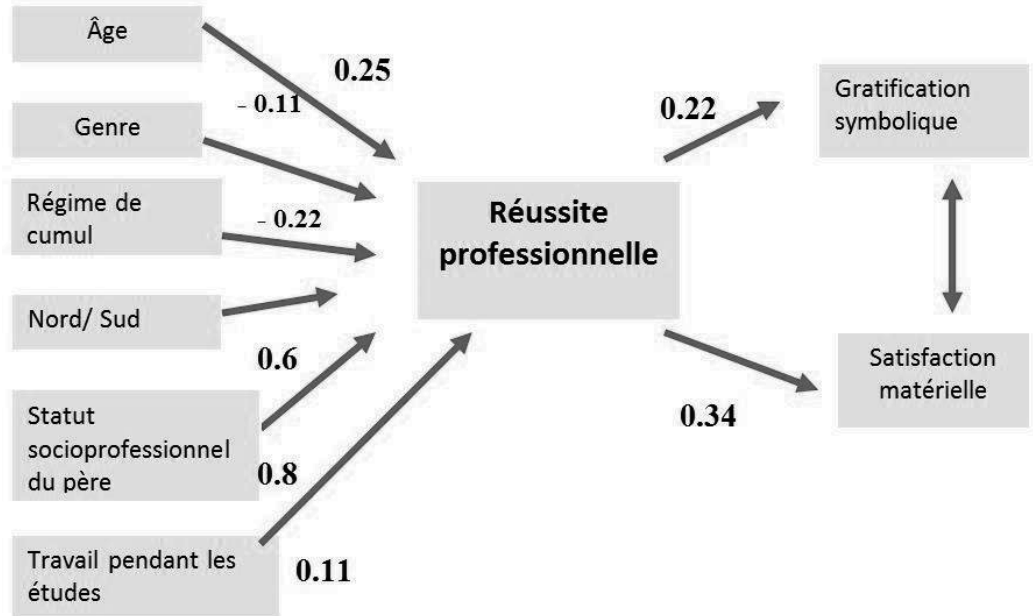

Ceci dit, les attributs sociodémographiques qui ont le plus contribué à l'explication d'une " carrière réussie " sont, par ordre d'importance, l'âge, comme il fallait s'y attendre puisque toute carrière a besoin de temps pour se développer; se consacrer en exclusivité à la profession libérale, reléguant à la marge de la profession les activités cumulées; le genre, au détriment des femmes; le fait d'avoir travaillé pendant les études, qui est confirmé en tant qu'ingrédient favorable à la réussite professionnelle mais qui est en déclin à l'heure actuelle; le statut socioprofessionnel du père, qui confirme le poids des origines sociales dans les carrières; et finalement être installé dans la région Sud, ce qui démontre l'importance de la dimension du marché.

De son côté, le succès professionnel contribue à expliquer les sentiments de gratification symbolique et de satisfaction matérielle. Toutefois, ces sentiments ne se recoupent pas totalement ( $r$ de Pearson= 39), étant donné la disjonction récurrente entre l'architecture comme profession, et 
l'architecture comme vocation, et, par ailleurs, la satisfaction matérielle se retrouve plus étroitement associée à la réussite professionnelle que la réalisation symbolique. Comme dans les professions artistiques, il existe en architecture la vocation sans la satisfaction matérielle correspondante, ce qui soutient statistiquement, une fois de plus, l'idée que l'éthos professionnel des architectes continue d'être divisé entre vocation et profession, et ce quelle que soit la dimension en cause.

\section{Conclusion}

Les résultats de l'enquête ont confirmé tout l'intérêt de la confrontation du binôme "vocation " et de "profession " chez Max Weber qui nous a, ainsi, permis de montrer que l'identité professionnelle des architectes se construit autour d'une " vocation" et d'un ethos esthétique social, qui s'épanouissent rarement de façon pleine, et d'un ethos technicoscientifique caractéristique d'une profession à base universitaire. Le renouvellement générationnel de l'architecture au Portugal, associé à l'ouverture de cours privés, constitue le grand changement ressenti au sein de ce groupe professionnel. Les architectes sont de plus en plus jeunes et le pourcentage de femmes ne cesse d'augmenter. De plus, les réseaux familiaux et sociaux ainsi que l'apprentissage en situation de travail pendant les études favorisent l'accès à la profession et la réussite professionnelle mais tendent à contribuer à la reproduction de l'ethos traditionnel en évitant la lutte des "nouveaux» et les " anciens ». De son côté, la réussite professionnelle est fortement associée à la "satisfaction matérielle " mais aussi à la "gratification symbolique ", même s'il existe entre les deux une dissociation qui confirme la centralité de la tension récurrente entre la "vocation " et "profession" en architecture telle comme elle est pratiquée aujourd'hui au Portugal. 


\section{Bibliographie}

BORGES, Vera (2009), Les comédiens et les troupes de théâtre au Portugal, Paris, L'Harmattan.

BOURDIEU, Pierre ([1979] 2003), La Distinction, Critique sociale du jugement, Paris, Les Éditions de Minuit.

CHAMPY, Florent (1998), Les architectes et la commande publique, Paris, PUF.

CHAMPY, Florent (2001), Sociologie de l'architecture. Coll. Repères, $n^{\circ} 314$, Paris, La Découverte.

DE MONTILERT, C. (1995), L'impossible autonomie de l'architecte. Sociologie de la production architecturale. Strasbourg: Presses Universitaires de Strasbourg.

EGBERT, Donald (1980), The Beaux-Arts Tradition in French Architecture, Princeton University Press.

FRANCASTEL, Pierre (1988), Art et technique aux XIXe et XXe siècles, Paris, Gallimard.

FREIDSON, Eliot (1986), "Les professions artistiques comme défi à I'analyse sociologique ". Revue française de sociologie, XXVII (3), pp. 431-443.

FREIDSON, Eliot. 1990. "Labours of love in theory and practice: a prospectus ». In The Nature of Work, Sociological Perspectives, eds K. Erikson, S.P. Vallas. New Haven: Yale University Press, 149-161.

KOSTOF, Spiro (ed.) (2000), Architect. Chapters in the History of the profession, Londres, University of California Press.

LARSON, Magali-Sarfati (1993), Behind the postmodern decade: architectural change in late twentieth-century Americ., Berkeley: University of California Press.

MENGER, Pierre-Michel (1999) "Artistic labor markets and careers". Annual Review of Sociology, 25, pp. 541-574.

MENGER, Pierre-Michel (2006), "Artistic labor markets: contingent work, excess supply and occupational risk management ", in Handbook of the Economics of Art and Culture, ed. V. A. Ginsburgh \& D. Throsby. Elsevier B. V., vol. 1 : 766-811.

MENGER, P. -M. (2009), Travail Créateur. S'Accomplir dans I'Incertain, Paris, Gallimard/ Seuil.

MENGER, P. -M. (2012), " Talent and inequalities: what do we learn from the study of artistic occupations? ", in Vera Borges et Pedro Costa, Criatividade $e$ Instituições. Os novos desafios aos artistas $e$ profissionais da cultura, Lisbonne, Imprensa de Ciências Sociais, pp. 49-75. 
MERTON, Robert (1988), " The Matthew effect in science, II, cumulative advantage and the symbolism of intellectual property ", Isis, 79, pp. 606-623.

MOULIN, Raymonde et al. (1973), Les architectes, métamorphose d'une profession libérale, Paris, Calmann-Lévy.

RAYNAUD, Dominique (2001), "Compétences et expertise professionnelle de l'architecte dans le travail de conception ". Sociologie du Travail, 43, pp. 451-469.

SIMON, Herbert (1981), As ciências do artificial, Lisboa, Editora Arménio Amado.

TAFURI, Manfredo (org.) (1972), Socialismo, città, architettur: URSS 1917-1937, Roma, Officina Edizioni.

WEBER, Max ([1904] 2002) L'éthique protestante et l'esprit du capitalisme, Paris, Plon.

WEBER, Max ([1919] 2005) Le savant et le politique: une nouvelle traduction: la profession et la vocation de savant, la profession et la vocation de politique, trad. et préface de Catherine Colliot-Thélène, Paris, Plon. 